\title{
Biological dosimetry of ionizing radiation: Evaluation of the dose with cytogenetic methodologies by the construction of calibration curves
}

\author{
Demetre Zafiropoulos ${ }^{*+}$, E. Facco ${ }^{\dagger}$ and Lucia Sarchiapone* \\ *Laboratori Nazionali di Legnaro - INFN, Legnaro (Pd), Italy \\ ${ }^{\dagger}$ Istituto Oncologico Veneto, Padova, Italy \\ ${ }^{\ddagger}$ zafiropoulos@lnl.infn.it
}

Published 1 September 2016

\begin{abstract}
In case of a radiation accident, it is well known that in the absence of physical dosimetry biological dosimetry based on cytogenetic methods is a unique tool to estimate individual absorbed dose. Moreover, even when physical dosimetry indicates an overexposure, scoring chromosome aberrations (dicentrics and rings) in human peripheral blood lymphocytes (PBLs) at metaphase is presently the most widely used method to confirm dose assessment. The analysis of dicentrics and rings in PBLs after Giemsa staining of metaphase cells is considered the most valid assay for radiation injury. This work shows that applying the fluorescence in situ hybridization (FISH) technique, using telomeric/centromeric peptide nucleic acid (PNA) probes in metaphase chromosomes for radiation dosimetry, could become a fast scoring, reliable and precise method for biological dosimetry after accidental radiation exposures. In both in vitro methods described above, lymphocyte stimulation is needed, and this limits the application in radiation emergency medicine where speed is considered to be a high priority. Using premature chromosome condensation (PCC), irradiated human PBLs (non-stimulated) were fused with mitotic CHO cells, and the yield of excess PCC fragments in Giemsa stained cells was scored. To score dicentrics and rings under PCC conditions, the necessary centromere and telomere detection of the chromosomes was obtained using FISH and specific PNA probes. Of course, a prerequisite for dose assessment in all cases is a dose-effect calibration curve. This work illustrates the various methods used; dose response calibration curves, with $95 \%$ confidence limits used to estimate dose uncertainties, have been constructed for conventional metaphase analysis and FISH. We also compare the doseresponse curve constructed after scoring of dicentrics and rings using PCC combined with FISH and PNA probes. Also reported are dose response curves showing scored dicentrics and rings per cell, combining PCC of lymphocytes and CHO cells with FISH using PNA probes after $10 \mathrm{~h}$ and $24 \mathrm{~h}$ after irradiation, and, finally, calibration data of excess PCC fragments (Giemsa) to be used if human blood is available immediately after irradiation or within $24 \mathrm{~h}$.
\end{abstract}

Keywords: Biological dosimetry; FISH-PNA probes; Premature chromosome condensation; PCC.

\section{Introduction}

Due to an increasing concern about accidental overexposures, as well as the threat of nuclear terrorism, the preparedness for medical management of radiation events is of great importance. ${ }^{1,2}$ In the event that physical dosimetry-based dose estimates are unavailable or a high-dose dosimeter response has yet to be confirmed, the analysis of

This is an Open Access article published by World Scientific Publishing Company. It is distributed under the terms of the Creative Commons Attribution 3.0 (CC-BY) License. Further distribution of this work is permitted, provided the original work is properly cited. 
induced chromosomal aberrations (CA) at metaphase in the lymphocytes of peripheral blood (PBL) is the most valid technique.,

In the case of a large number of victims and high levels of radiation exposure, the time required for dose estimation is important for medical triage. Scoring of unstable CA, such as dicentric chromosomes (dicentrics), centric rings and fragments at metaphases in PBL after Giemsa staining, is presently the gold standard technique for biological dosimetry. Analysis of dicentrics with Giemsa-stained samples requires a well-trained and skilled observer ${ }^{5}$ due to inherent difficulties in the method. ${ }^{6}$

As the precise quantification of unstable $\mathrm{CA}$ is crucial for estimation of the absorbed dose, the Fluorescence in Situ Hybridization (FISH) technique can replace the conventional Giemsa staining. The method is based on the use of fluorescent probes to visualize chromosomal abnormalities with an increase of signal intensity, a reduction in the time needed to obtain the results and the possibility of simultaneous detections of various regions of interest. Recently, the application of the FISH technique by means of telomere and centromere peptide nucleic acid (PNA) probes has proven to become a candidate for the new standard method for biological dosimetry in radiation biology: it is able to shorten hybridization time $(12 \mathrm{~h})$, it has a low cost, and it has high affinity and specificity to the target DNA. ${ }^{7}$

Unfortunately, Giemsa staining and FISH methodologies using metaphase chromosomes require a two-day culture needed for lymphocytes to reach mitosis - a major drawback for the use of the method in case of radiation emergency, since a rapid and accurate estimation of the dose is a high priority. As an alternative, one can perform cell fusion of human lymphocytes with the mitotic cells of Chinese hamster ovary (CHO) using the chemical agent polyethylene glycol (PEG) and inducing premature chromosome condensation (PCC), as reported elsewhere. ${ }^{8,9}$ In such a way, it is possible to visualize chromosome damage directly in non-stimulated lymphocytes without the need for the two-day culture of blood samples. This technique allows a shortened processing time of $3 \mathrm{~h}$ instead of the $50 \mathrm{~h}$ otherwise required. The quantification of exposure using PCC is limited to the analysis of chromosome fragments and rings, since staining with Giemsa of prematurely condensed chromosomes does not allow visualization of the centromeric regions and, consequently, the identification of dicentrics, centric rings and acentric fragments.

This contribution presents an improved method for a rapid and accurate detection of dicentric chromosomes in non-stimulated lymphocyte PCCs using the FISH technique and PNA probes for the identification of chromosome centromeres and telomeres. The detection of centromere and telomere signals leads to a precise scoring not only of dicentrics but also of all $\mathrm{CA}$, with a better characterization of acentric chromosomes.

To assess the liability of the PCC methodology, the dose estimation capability was compared with the dose estimates of the methodologies described above. Using in vitro experiments, we have constructed calibration curves for all the above methodologies for dose estimation purposes, where unstable CA are plotted against gamma dose given (dose-effect). 
The gamma dose interval investigated was between 0.3 Gy and 8 Gy and more than $12.5 \times 10^{3}$ cells in metaphase or interphase were scored using microscopic analysis of chromosome aberrations. Dose-response calibration curves, with 95\% confidence limits used to estimate uncertainties, have been constructed for conventional metaphase analysis and FISH; the curves are linear quadratic, and for their construction, a minimum of 500 cells or 100 dicentrics per dose were analyzed. Only metaphases with 46 chromosomes were taken into account. The Poisson distribution of the CA was tested for every dose and the coefficients of the linear quadratic equation, their errors and $\mathrm{p}$ value were calculated. At present, no dose assessment of irradiated blood samples with unknown doses and based on the calibration curves reported have been performed.

Finally, in order to test the goodness of the constructed calibration curves for triage biodosimetry, whole blood was irradiated with two different doses of 1 Gy and 3 Gy of gamma irradiation. The samples were then coded blindly, and the above methodologies were performed. The yields of dicentrics plus rings Giemsa stained and FISH with PNA probes, excess PCC fragments Giemsa stained or dicentrics plus centric rings in lymphocyte PCCs visualized by the FISH technique with PNA probes were obtained scoring 30 and 50 cells.

\section{Materials and Methods}

\subsection{Culture of $\mathrm{CHO}$ cells and human lymphocytes for conventional metaphase analysis (DCA, FISH)}

\subsubsection{Cell cultures and irradiation conditions}

Chinese hamster ovary $(\mathrm{CHO})$ cells were grown in McCoy's 5A culture medium supplemented with $10 \%$ fetal calf serum, $1 \%$ L-Glutamine, and $1 \%$ penicillinstreptomycin antibiotics. The cells were incubated $24 \mathrm{~h}$ at $37^{\circ} \mathrm{C}$ in a humidified atmosphere containing $5 \% \mathrm{CO}_{2}$ and maintained as exponentially growing monolayer cultures in $75 \mathrm{~cm}^{2}$ flasks at an initial density $6 \times 10^{5}$ cells/flask. Then in order to collect the maximum number of mitotic cells, the culture medium was changed with fresh McCoy's $5 \mathrm{~A}$ medium containing $1 \%$ colcemid solution and incubated for four hours. Mitotic cells were obtained by a standard selective detachment procedure using a Trypsin/Edta solution and transferred to a culture tube, ready to perform PCC.

Peripheral blood from three healthy informed and consensual individuals was drawn in heparinized tubes. Every sample was set up by adding $0.5 \mathrm{ml}$ of whole blood to $5 \mathrm{ml}$ of medium (RPMI Medium 1640), 10\% fetal calf serum, 1\% of Phytohemagglutinin (PHA), $1 \%$ L-Glutamine, $1 \%$ penicillin-streptomycin antibiotics. Cultures were then incubated in a humidified atmosphere at $37^{\circ} \mathrm{C}$ with $5 \% \mathrm{CO}_{2}$ for $48 \mathrm{~h} ; 50 \mu \mathrm{l}$ of colcemid was added to the cultures and maintained four hours. Cells were then treated with hypotonic $\mathrm{KCl}$ (75 $\mathrm{mM}$ ) and fixed with Carnoy's Fixative (3 methanol: 1 glacial acetic acid) two to three times. After being centrifuged, the chromosome spreads were prepared manually as follows: one or two drops of fixative were added to dilute the pellet, and one or two drops 
(about $20 \mu \mathrm{l}$ each) of pellet were dropped on wet slides, air dried, and stained with $3 \%$ of Giemsa. Slides were ready to be observed using light microscopy coupled with an image analysis system (MetaSystems, Germany) and scored for CA. Slides that had to be processed with FISH (no Giemsa staining) were aged for at least $24 \mathrm{~h}$ at $37^{\circ} \mathrm{C}$. Irradiation of all samples was carried out in a ${ }^{60} \mathrm{Co}$ Gamma Beam irradiator at room temperature and with a dose rate of $0.5 \mathrm{~Gy} / \mathrm{min}$ at $30 \mathrm{~cm}$. Different irradiation times were used to cover the range from $0.3-8$ Gy. All blood samples after irradiation and before culture with the method described above were kept at $37^{\circ} \mathrm{C}$ for $2 \mathrm{~h}$.

\subsubsection{FISH with PNA probes for centromeric and telomeric staining}

FISH is a molecular technique that helped improve the resolving power of classical cytogenetics to analyze chromosome aberration. In our experiments, we used telomeric and centromeric PNA probes to highlight the presence of dicentric or multicentric chromosomes, rings, fragments of DNA.

The protocol is based on a series of steps; the initial series is designed to clean and fix the chromosomal metaphases on the slide. To fix the metaphase, the slides were immersed in a 4\% formaldehyde in phosphate-buffered saline (PBS) solution for $10 \mathrm{~min}$, quickly washed in PBS, immersed in a digest pepsin solution $\left(1 \mathrm{mg} / \mathrm{ml}\right.$ pepsin in $\mathrm{H}_{2} \mathrm{O}$ and $\mathrm{HCl} 37 \%$ ) for $10 \mathrm{~min}$, washed in fresh PBS, fixed again and then dehydrated in 70, 90 and $100 \%$ ethanol for $5 \mathrm{~min}$. Once the slides were dry and the ethanol evaporated, the codenaturation step took place in which at the same time the molecular PNA probes and the DNA on the slides were denatured. The slides were then treated with $20 \mu \mathrm{l}$ of probe solution (preparation of 70\% formamide, 1\% 1M Tris buffered saline, 5\% NEN Blocking Reagent in maleic solution $10 \%, 9 \% \mathrm{MgCl}_{2}$ buffer, $2 \% 25 \mu \mathrm{g} / \mathrm{ml}$ TelC-FAM, and 5\% 25 $\mu \mathrm{g} / \mathrm{ml}$ Cent-Cy3-Panagene, South Korea) and co-denatured for $3 \mathrm{~min}$ at $80^{\circ} \mathrm{C}$. Slides were put in a humidified chamber at $4^{\circ} \mathrm{C}$ overnight. In the third step, in order to remove excess probe and what was hybridized non-specifically, stringency washes of the slides were performed $(70 \%$ formamide solution two times for 15 minutes each, TBS/Tween $0.08 \%$ solution for three times and then dehydration in 70,90 and $100 \%$ ethanol). After washing, the slides were counter-stained with VECTASHIELD Mounting Medium with DAPI (Vector Laboratories) and mounting medium. Chromosome spreads were analyzed manually with Zeiss Axio Imager Z2 microscope provided with fluorescence and software MetaSystems ISIS 5.5.

\subsection{Premature chromosome condensation}

\subsubsection{Isolation of lymphocytes}

Lymphocytes were isolated from heparinized peripheral blood using the Ficoll-Paque method and following the procedures suggested by the manufacturer. In a $14 \mathrm{ml}$ tube, we added $5 \mathrm{ml}$ of Biocoll Separating Solution, and we layered carefully and gently $5 \mathrm{ml}$ of whole blood without any dilution using a $5 \mathrm{ml}$ pipette, so that we did not disturb the interphase between the separating solution and the whole blood (i.e. avoid any mixing). 
After centrifugation at $1800 \mathrm{rpm}$ for $20 \mathrm{~min}$, the lymphocytes were concentrated at interphase of the gradient, collected with a $5 \mathrm{ml}$ pipette, and diluted in $10 \mathrm{ml} \mathrm{RPMI}[+]$ HEPES culture medium with serum. The lymphocytes were then centrifuged at $1500 \mathrm{rpm}$ for $10 \mathrm{~min}$, the cell pellet was re-suspended in RPMI full medium, and the lymphocytes were ready to be used for the different experimental points. Depending on the number of experimental points that will be considered, an appropriate number of $\mathrm{CHO}$ mitotic cells must be prepared for cell fusion as described in Sec. 2.1.1. Indicatively, lymphocytes obtained from $1 \mathrm{ml}$ of blood are sufficient for 1-2 experimental points.

\subsubsection{Fusion of $\mathrm{CHO}$ mitotics with isolated peripheral blood lymphocytes}

In a $14 \mathrm{ml}$ round-bottomed culture tube, lymphocytes were mixed and re-suspended in a medium without serum, with mitotic $\mathrm{CHO}$ cells also re-suspended in a medium without serum containing colcemid. The whole mixture was then centrifuged for 6 min so that the mixed cells in the pellet were not too packed at a medium value of rpm (e.g. $1000 \mathrm{rpm}$ ). The supernatant was poured off while always keeping the tube inverted. Once blotted on a paper towel, the tube was placed upside down in the lined test tube rack (this prevents the residual drop of medium from covering the cell pellet); the tube was reblotted until cells to be fused had a minimum fluid. After a few minutes, all while keeping the tube inverted, a $200 \mu \mathrm{l}$ pipette was used to inject, at room temperature $\left(20^{\circ} \mathrm{C}\right), 150 \mu \mathrm{PEG}$ (Polyethylene glycol 1500, readymade solution: PEG 50\% w/v, $75 \mathrm{mM}$ Hepes) into the tube straight into the cell pellet. At this point, the tube was immediately turned upright and placed in the test tube rack without shaking it. The task was to inject PEG solution directly into cell membranes, avoiding any fluid between the cells and PEG (that is why the tubes were kept inverted until the addition of PEG). At this point, the cell pellet appeared detached from the tube bottom forming clumps in the PEG solution (for this purpose we used low rpm centrifugation and round-bottom tubes instead of conical ones). We point out that if the pellet remains attached to the bottom, the fusion will not succeed.

PBS or RPMI without serum $(1.5 \mathrm{ml})$ was then slowly added to the tubes and mixed with the PEG solution by gently shaking so that the solution was homogeneous with small aggregations of $\mathrm{CHO}$ cells with lymphocytes. After being centrifuged for 6 min at $1000 \mathrm{rpm}$, the supernatant was poured off, blotted, and $0.7 \mathrm{ml}$ of pre-warmed RPMI growth medium was added, containing colcemid to hold the $\mathrm{CHO}$ cells at metaphase. The medium was added very slowly by tapping the tube gently so that $\mathrm{CHO}$ cells with lymphocytes remained suspended in small aggregations. If the pellet breaks into a single cell suspension, cell fusion will succeed. To optimize cell fusion, $0.7 \mathrm{ml}$ of complete lymphocyte growth medium containing PHA was used. ${ }^{9}$ Finally, the tubes were placed in a test tube rack and incubated at $37^{\circ} \mathrm{C}$ for $75 \mathrm{~min}$. At this point good fusion and premature chromosome condensation in lymphocytes was completed, so 46 distinguished single chromatid chromosomes in control lymphocytes were visualized. Subsequently, chromosome preparations were obtained using the standard procedures already reported on: Hypotonic $\mathrm{KCl}(0.075 \mathrm{M})$ and Carnoy's fixative (1:3 v/v acetic acid/methanol). The 
air-dried chromosomes spreads on slides were stained with $3 \%$ Giemsa, and the FISH technique with PNA probes was also applied to a part of the air dried slides.

The fusion with CHO cells following FISH was applied after waiting $10 \mathrm{~h}$ and $24 \mathrm{~h}$ after the irradiation. For both, the number of unstable CA (dicentrics plus central rings) was scored as a function of the gamma dose delivered. The gamma doses given in the two cases were 1, 3, 5, 7 Gy for the curve obtained after $10 \mathrm{~h}$ and 1, 2, 4, 6, 8 Gy for the one after $24 \mathrm{~h}$.

\subsection{Statistical analysis}

The dose-effect relationship and the $\mathrm{p}$ value for the $\alpha$ and $\beta$ coefficients were determined for dicentric chromosomes and centric rings fitting the frequencies with a linear quadratic method using software SigmaPlot version 13.0. The $u$ test of the goodness of the fit was used to evaluate the yield of the unstable aberrations already mentioned with dose for the Poisson distribution; ${ }^{3}$ this relies on the value of the dispersion index $\left(\sigma^{2} / y\right)$, which provides a measure of the correspondence of the sample's variance with that predicted by the Poisson distribution. ${ }^{10}$

The formula used to calculate the standard $u$ test was:

$$
u=\left[\left(\sigma^{2} / \mathrm{y}\right)-1\right] \text { sqrt N-1/2(1-1/X), }
$$

where $\sigma^{2}$ is the variance, $\mathrm{y}$ is the mean, $\mathrm{N}$ is the number of cells analyzed and $\mathrm{X}$ is the number of observed aberrations. With $u$ values included between +1.96 and -1.96 , the aberrations are considered to be Poisson distributed. Once the dose-effect relationship is established, the uncertainty expression in a measured yield of CA is given in terms of $95 \%$ of confidence limits. In the construction of these curves, Merkle's approach ${ }^{11}$ was adopted. For excess PCC fragments (Giemsa staining) linear dose-effect curves were obtained.

\section{Results and Discussion}

In order to achieve a retrospective dose assessment capability, gamma ray CA dose-effect calibration curves were established in our laboratory for all the cytogenetic methods applied. All samples were taken from three healthy donors, and the mean values and associated standard deviations reported in the figures are calculated from three independent experiments.

\subsection{Dicentric chromosome assay (DCA) using Giemsa staining}

With what concerns the gold standard method, non-irradiated chromosomes in metaphase are shown in Fig. 1; the irradiated ones are shown in Fig. 2, reporting dicentrics, acentric fragments and centric ring. 


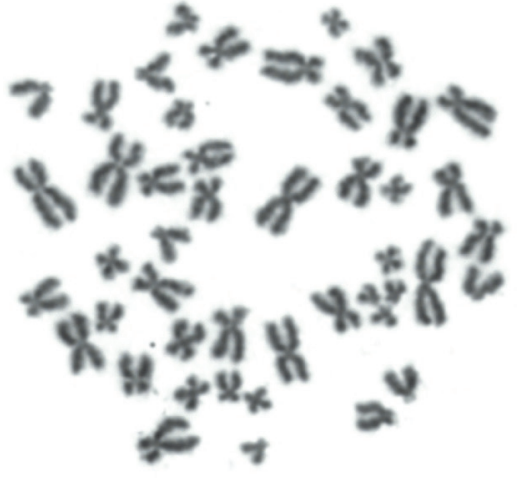

(A)

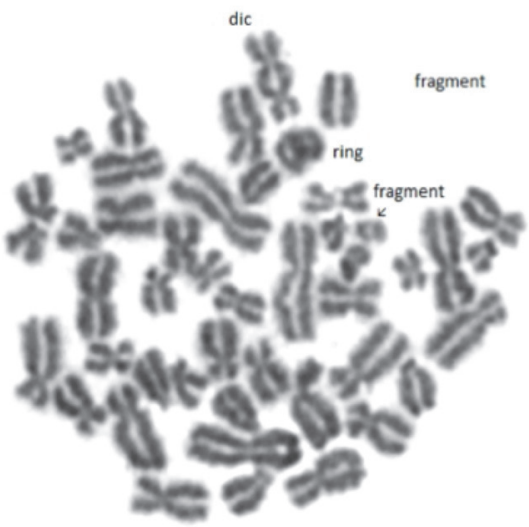

(B)

Fig. 1. (A) Metaphase cell with 46 normal chromosomes (not irradiated) and (B) aberrant metaphase cell irradiated with 4 Gy gamma-rays showing a dicentric chromosome (dic), a centric ring (ring) and acentric fragments.

After gamma ray exposure with doses ranging from $0.3-5 \mathrm{~Gy}$, a total of 6070 metaphases were manually analyzed, revealing $953 \mathrm{CA}$ (tricentric chromosomes were scored as two dicentrics). The trend of the CA frequency increased with the dose ranging from 0.014 at 0.3 Gy to 1.71 at $5 \mathrm{~Gy}$, and the distribution follows the Poisson distribution with $u$ values between \pm 1.96 . The background aberration frequency was $0.8 \mathrm{CA}$ for 1000 cells, a value that is perfectly consistent with other published data. ${ }^{3-12}$ Table 1 reports the distribution of the $\mathrm{CA}$ in cells irradiated with gamma rays, the $u$ values, and the dispersion factor of dicentrics and centric rings.

Table 1. DCA: The number of CA (dicentrics: dic, centric rings: $\mathrm{cR}$ ) in Giemsa-stained metaphase cells, their distribution (1-6 CA/cell), $u$ values and dispersion index factors $\left(\sigma^{2} / \mathrm{y}\right)$ are summarized from three different experiments (three donors).

\begin{tabular}{|c|c|c|c|c|c|c|c|c|c|c|c|}
\hline $\begin{array}{l}\text { Dose } \\
\text { (Gy) }\end{array}$ & $\begin{array}{c}\text { Metaphases } \\
\text { scored }\end{array}$ & $\mathrm{CA}$ & 0 & 1 & 2 & 3 & 4 & 5 & 6 & $\sigma^{2} / \mathbf{y}$ & $u$ \\
\hline 0 & 2468 & 2 & 2466 & 2 & & & & & & 1.00 & -0.02 \\
\hline 0.3 & 994 & 14 & 980 & 14 & & & & & & 0.99 & -0.30 \\
\hline 0.5 & 540 & 16 & 524 & 16 & & & & & & 0.97 & -0.47 \\
\hline 0.7 & 404 & 22 & 382 & 22 & & & & & & 0.95 & -0.76 \\
\hline 1 & 500 & 53 & 448 & 51 & 1 & & & & & 0.97 & -0.47 \\
\hline 2 & 501 & 155 & 368 & 116 & 14 & 2 & & 1 & & 1.09 & 1.38 \\
\hline 3 & 291 & 156 & 176 & 80 & 30 & 4 & 1 & & & 1.08 & 1.00 \\
\hline 4 & 175 & 197 & 47 & 76 & 40 & 9 & 1 & 2 & & 0.82 & -1.65 \\
\hline 5 & 198 & 338 & 30 & 59 & 64 & 36 & 3 & 5 & 1 & 0.81 & -1.93 \\
\hline
\end{tabular}


The dose effect curve, shown in Fig. 2 (black curve), is based on the results obtained from Table 1 . The curve is analyzed for the goodness of fit with a linear-quadratic model $\mathrm{f}=\mathrm{C}+\alpha \mathrm{D}+\beta \mathrm{D}^{2}$. The calculated $\alpha$ and $\beta$ coefficients are in agreement with previously published ones. ${ }^{3,13,14}$ Figure 2 also shows the 95\% upper and lower confidence levels curves. For all the curves reported in Fig. 2, the coefficient of determination $\mathrm{R}^{2}$ was equal to 0.99 .

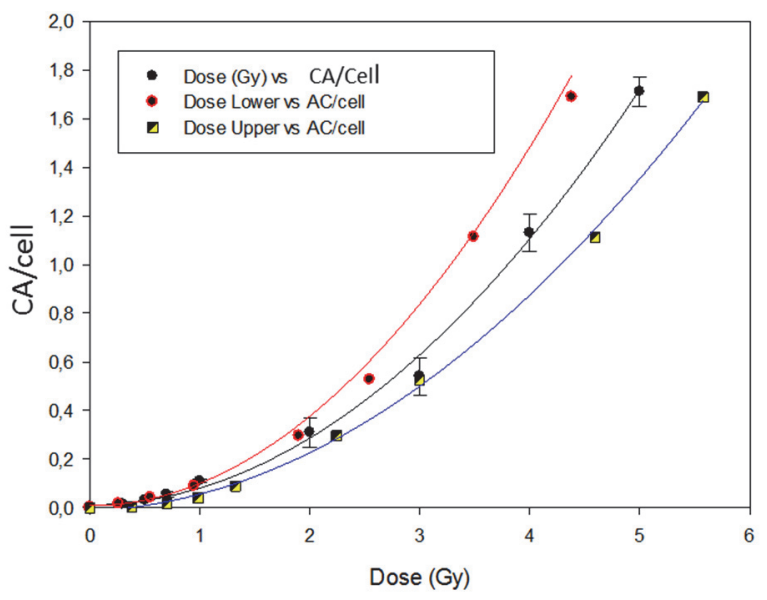

Fig. 2. Linear quadratic dose-effect curves of CA yield (black) with 95\% upper and lower confident limits (red and blue curves) used to evaluate the uncertainty of the given dose. Error bars represent standard deviations of mean CA frequencies calculated from three independent experiments.

\section{I) DCA using FISH with centromere/telomere PNA probes}

The usefulness of FISH analysis with pan-telomere and pan-centromere PNA probes in biological dosimetry has already been shown, especially for blood samples exposed to high doses. ${ }^{5}$ In particular, in the presence of multicentric chromosomes (tricentrics, tetracentrics, etc.), their detection is more accurate, resulting in a better dose evaluation as shown in Fig. 3(A). This is due to the lack of complications in distinguishing centromeres from the crossing of long chromosomes. Moreover, the detection of rings is easy to achieve, while in Giemsa, they could be missed even by a well-trained observer. Furthermore, false positive or negative dicentrics are easy to detect using DCA with FISH and PNA probes if compared with the Giemsa staining method as shown in Fig. 3(B). 


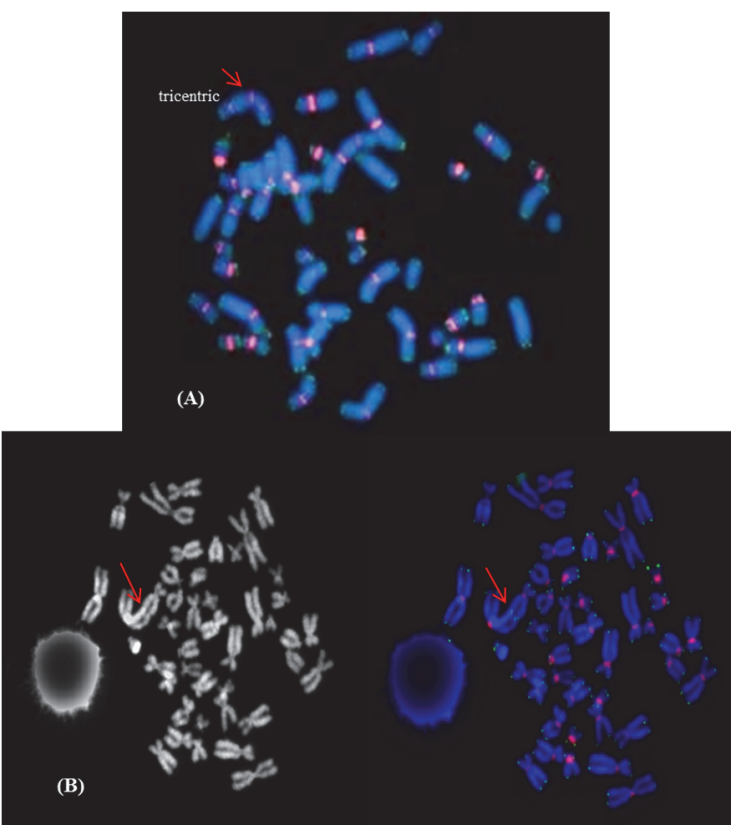

Fig. 3. (A): Accurate detection of dicentric and tricentric chromosomes with FISH analysis (4 Gy of ${ }^{60} \mathrm{Co}$ gamma's). (B): An example of false positive detection - the indicated chromosome recognizable as dicentric in $\mathrm{b} / \mathrm{w}$ is easily identified as two different chromosomes due to the presence of the telomeres (green signals).

Also for the FISH technique, using telomere/centromere PNA probes, we estimate in vitro the unstable CA per cell as a function of the delivered gamma dose. More than 4500 metaphases have been analyzed manually with the microscope under fluorescence, and the results are presented in Table 2. The same table also reports the distribution of the CA in cells irradiated with gamma rays, the $u$ value and the dispersion factor of dicentrics and centric rings. Dose-effect calibration curves, including the 95\% upper and lower confidence level curves, are shown in Figs. 4(A) and 4(B). For all reported curves, the coefficient of determination $\mathrm{R}^{2}$ was better than 0.99 .

Table 2. FISH+PNA probes: number of CA (dic and $\mathrm{cR}$ ), their distribution in the cells, $u$ values, and dispersion index factors $\left(\sigma^{2} / \mathrm{y}\right)$ are summarized from three different experiments.

\begin{tabular}{|c|c|c|c|c|c|c|c|c|c|c|c|c|}
\hline $\begin{array}{l}\text { Dose } \\
\text { (Gy) }\end{array}$ & Metaphases & $\begin{array}{c}\text { Num } \\
\text { CA }\end{array}$ & 0 & 1 & 2 & 3 & 4 & 5 & 6 & 7 & $\sigma^{2} / y$ & $u$ \\
\hline 0 & 1942 & 4 & 1938 & 4 & & & & & & & 1.00 & -0.06 \\
\hline 0.5 & 615 & 19 & 596 & 19 & & & & & & & 0.97 & -0.53 \\
\hline 0.7 & 540 & 32 & 508 & 32 & & & & & & & 0.94 & -0.96 \\
\hline 1 & 520 & 69 & 457 & 57 & 6 & & & & & & 1.04 & 0.70 \\
\hline 2 & 373 & 160 & 244 & 104 & 19 & 6 & & & & & 1.04 & 0.50 \\
\hline 3 & 202 & 168 & 85 & 75 & 32 & 7 & 2 & & & & 0.94 & -0.58 \\
\hline 4 & 182 & 251 & 46 & 68 & 35 & 22 & 9 & 1 & 1 & & 1.06 & 0.58 \\
\hline 5 & 152 & 296 & 17 & 51 & 36 & 31 & 8 & 8 & & 1 & 0.95 & -0.46 \\
\hline
\end{tabular}




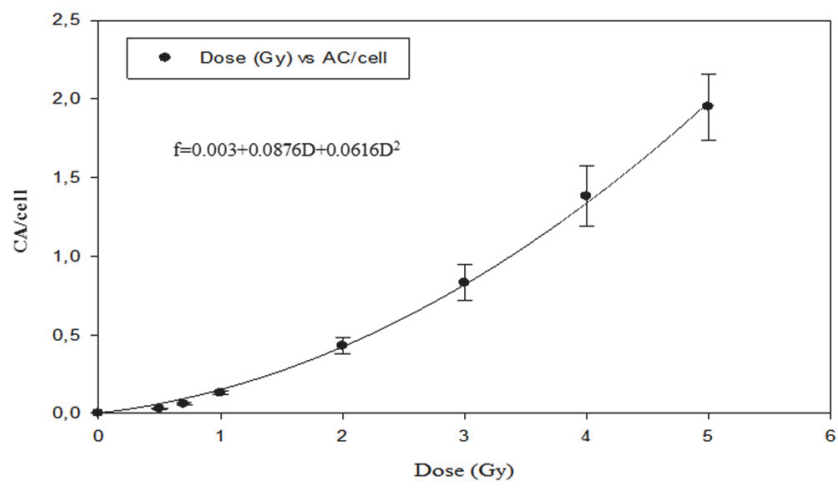

(A)

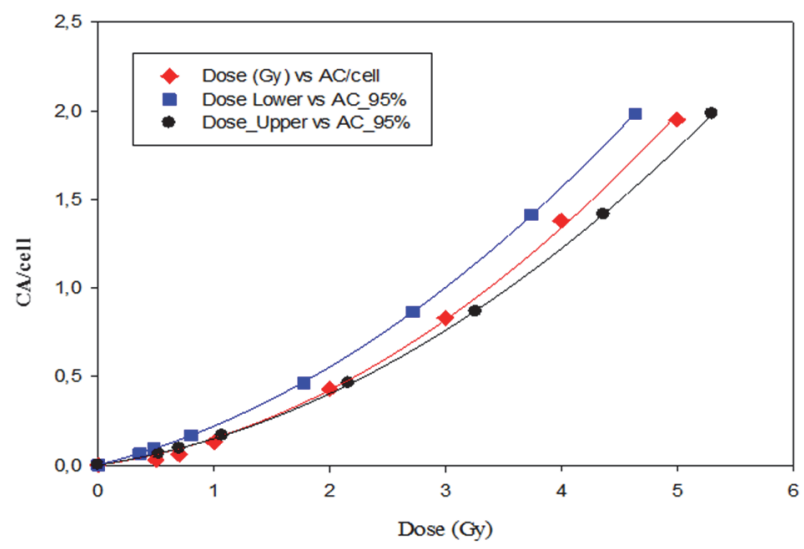

(B)

Fig. 4. (A): FISH+PNA probes - Linear quadratic dose-effect curves of CA yield. Mean \pm SD values are calculated from three independent experiments. (B): FISH+PNA probes - Linear quadratic dose-effect curves of CA yield (red) with 95\% upper and lower confident limits (blue and black curves) used to evaluate the uncertainty of the given dose; $f=C A$ frequency, $D=$ dose $(G y)$.

The calibration curves constructed with the DCA Giemsa staining and using FISH with centromere/telomere PNA probes chromosomes are plotted in Fig. 5, and a comparison of the sensitivity of the two methodologies can be made. It is evident that in the evaluation of the chromosomic aberrations, a better sensitivity is achieved using the FISH technique and chromosomes telomere/centromere painting with PNA probes. In particular, for doses greater than $1 \mathrm{~Gy}$, the estimation of the irradiated dose by FISH analysis seems to be more accurate than that by the conventional Giemsa analysis. The number of CA scored with FISH and PNA probes at 5 Gy gamma dose is in good agreement with the dicentric frequency using FISH and PNA probes at the same dose reported elsewhere. ${ }^{5}$ 


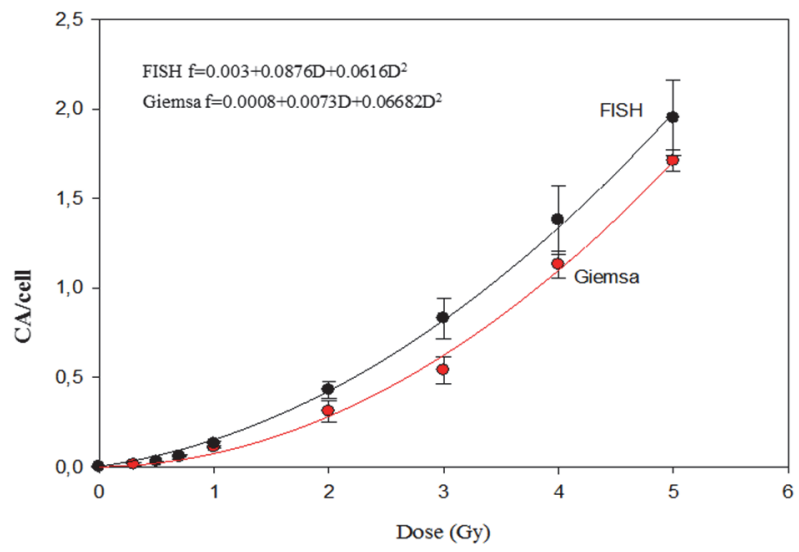

Fig. 5. Dose-effect relationship of dicentric and centric ring formation based on evaluation of cytogenetic slides after Giemsa-staining and FISH with PNA probes for centromeres and telomeres, respectively. Error bars represent standard deviations from mean $\mathrm{CA}$ frequencies calculated from three independent experiments. $\mathrm{f}=$ CA frequency, $\mathrm{D}=$ dose $(\mathrm{Gy})$.

\section{II) Chromosome aberrations estimation using PCC in interphase cells}

The methodologies described above need the lymphocytes stimulation to start the cell cycle and allow us to analyze unstable CA at the first metaphase. This implies a time of at least $50 \mathrm{~h}$ before any scoring of the first metaphases and evaluation of the dose in case of an accident. It is of prime importance, especially in radiation emergency medicine, to reduce the time required for dose response.

Premature chromosome condensation of human lymphocytes with mitotic CHO cells, as previously described, allows in $3 \mathrm{~h}$ (without any stimulation) a direct observation of primary DNA damage induced in interphase nuclei from ionizing radiation detected as excess fragments. Quantification of the exposure using PCC, a unique sensitive method to evaluate chromosomal damage immediately after exposure, ${ }^{8}$ was for many years applied in the analysis of chromosomic fragments. The construction of an appropriate calibration curve presupposes the analysis of chromosome fragments produced after irradiation of PBL at different gamma doses and after fusion with mitotic $\mathrm{CHO}$ cells (see Fig. 6).

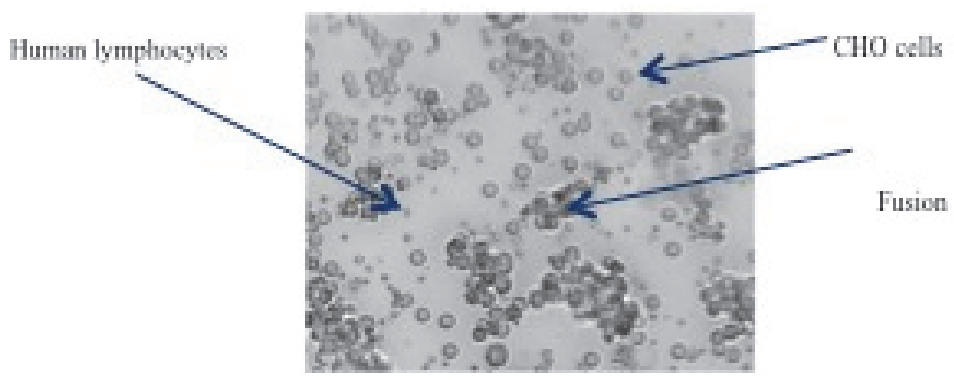

Fig. 6. Fusion process of human lymphocytes with $\mathrm{CHO}$ mitotic cells. 
Due to the recombination of the fragments over time, two different calibration curves were constructed. In the first curve, fusion occurred immediately after irradiation, and the excess chromosomal fragments (more than 46) are reported with the dose given. In the second curve, fusion took place $24 \mathrm{~h}$ after irradiation. Figures 7(A) and 7(B) show microscope images. The chromosomic fragments can be counted very easily by using dedicated acquisition software (Metasystems, Germany). In the case of the control sample, the excess fragments (considering 46 chromosomes), as an average, are considered to be equal to zero. The results after the calibration curves construction are shown in Figs. 8(A) and 8(B).

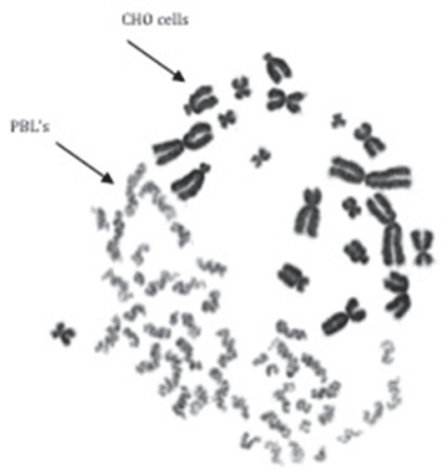

(A)

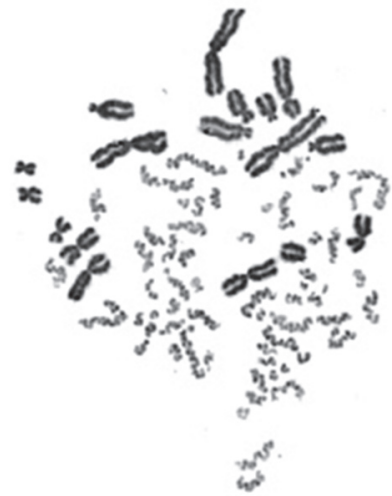

(B)

Fig. 7. (A): Human non irradiated lymphocytes fused with mitotic CHO cells; (B): Human lymphocytes irradiated with 5 Gy gamma and fused with mitotic $\mathrm{CHO}$.

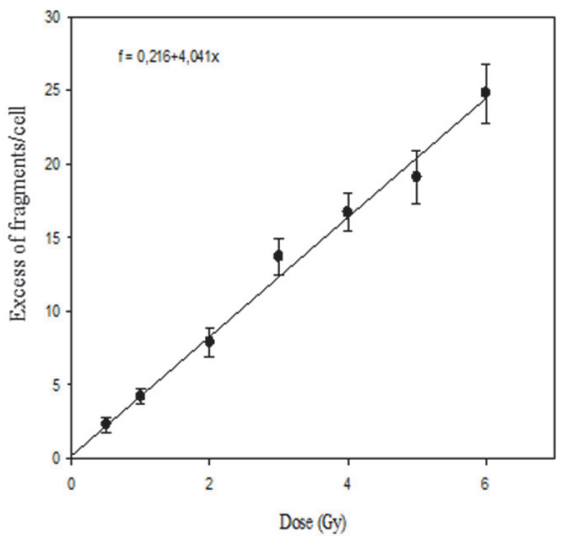

(A)

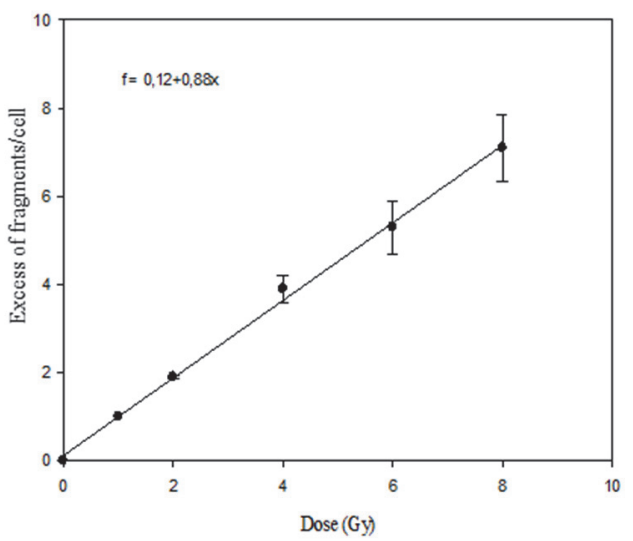

(B)

Fig. 8. (A): Human Lymphocytes fused immediately after irradiation; (B): Human lymphocytes fused after $24 \mathrm{~h}$ from irradiation. $\mathrm{f}=$ excess fragments yield, $\mathrm{x}=$ dose $(\mathrm{Gy})$. 
III) Chromosome aberrations estimation using PCC in interphase cells after telomere/centromere staining with PNA probes

The advantage of the PCC technique as a useful dosimetric tool for obtaining results without the need of human lymphocyte stimulation is lessened by the fact that dicentrics after irradiation cannot be visualized. This limitation is due to the specific morphology of interphase condensed chromosomes and the inability to detect centromeric regions after uniform staining. ${ }^{15}$

In order to reassess the limitation of the technique, we propose an improved method based on the capability to apply the FISH technique using PNA probes for telomere and centromere chromosome staining. We can efficiently detect dicentric or multicentric chromosomes indicating clear and separate centromeres in a single prematurely condensed chromosomal structure. Figure 9 shows the detection of prematurely condensed dicentric chromosomes in fused cells using FISH and PNA probes. Red arrows indicate dicentric chromosomes induced by 3 Gy gamma irradiation by a ${ }^{60} \mathrm{Co}$ source.

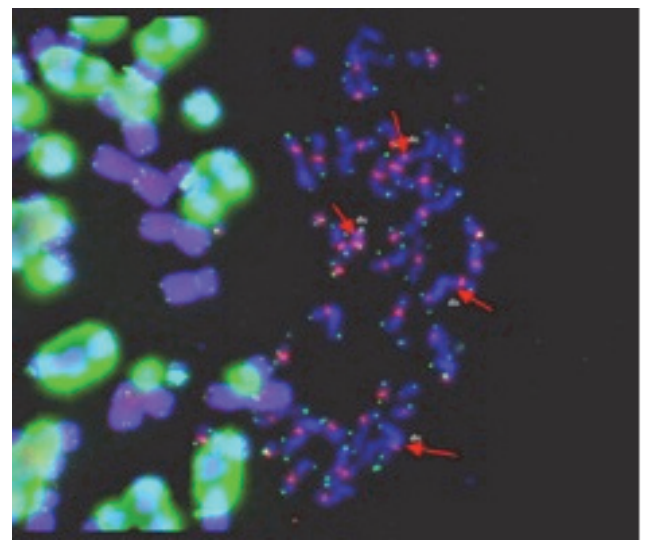

Fig. 9. Human irradiated lymphocytes fused with mitotic $\mathrm{CHO}$ cells (3 Gy gamma dose). Dicentric chromosomes are indicated by the red arrows.

Once the method was consolidated, taking into account that we can detect dicentrics in an accurate and rapid way, we considered the opportunity to verify the kinetics of the formation of the dicentrics proceeding with the fusion of PBL with mitotic $\mathrm{CHO}$ cells 10 and $24 \mathrm{~h}$ after lymphocyte irradiation. In such a way, we are able to ascertain if the fusion after 10 or $24 \mathrm{~h}$ after irradiation influences the formation of dicentrics chromosomes.

The number of metaphases, CA distribution in cells, and $u$ values with index dispersion are reported in Tables 3 and 4 . In the case of Table 3, lymphocytes are irradiated with gamma doses from 1 to $7 \mathrm{~Gy}$ and fused after $10 \mathrm{~h}$ from irradiation with mitotic CHO cells. At least 1000 cells in interphase (controls included) were analyzed manually. The number of cells scored for every dose was at least 500 or 100 dicentrics. Similarly, in Table 4 lymphocytes are irradiated with gamma doses from 1 to 8 Gy and fused after $24 \mathrm{~h}$ from irradiation with mitotic $\mathrm{CHO}$ cells. 
Table 3. Cytogenetic results obtained from three different samples of blood irradiated with ${ }^{60} \mathrm{Co} \gamma$-rays up to $7 \mathrm{~Gy}$. Fusion with PCC technique was performed at $10 \mathrm{~h}$ after irradiation. Centromere and telomere staining was performed using FISH-PNA probes.

\begin{tabular}{|c|c|c|c|c|c|c|c|c|c|c|c|c|}
\hline $\begin{array}{l}\text { Dose } \\
\text { (Gy) }\end{array}$ & Metaphases & Num CA & 0 & 1 & 2 & 3 & 4 & 5 & 6 & 7 & $\sigma^{2} / y$ & $u$ \\
\hline 0 & 521 & 0 & 521 & & & & & & & & 0.00 & 0.00 \\
\hline 1 & 288 & 104 & 196 & 80 & 12 & & & & & & 0.87 & -1.53 \\
\hline 3 & 99 & 105 & 32 & 37 & 24 & 4 & 2 & & & & 0.86 & -0.97 \\
\hline 5 & 43 & 100 & 3 & 6 & 16 & 13 & 3 & 1 & 1 & & 0.65 & -1.61 \\
\hline 7 & 72 & 196 & 4 & 9 & 21 & 22 & 7 & 5 & 2 & 2 & 0.85 & -0.89 \\
\hline
\end{tabular}

Table 4. Cytogenetic results obtained from three different samples of blood irradiated with up to 8 Gy. Fusion with PCC technique was performed at $24 \mathrm{~h}$ after irradiation. Centromere and telomere staining was performed using FISH-PNA probes.

\begin{tabular}{|c|c|c|c|c|c|c|c|c|c|c|c|c|c|}
\hline $\begin{array}{l}\text { Dose } \\
\text { (Gy) }\end{array}$ & Metaphases & Num CA & 0 & 1 & 2 & 3 & 4 & 5 & 6 & 7 & 8 & $\sigma^{2} / y$ & $u$ \\
\hline 0 & 500 & 0 & 500 & 0 & & & & & & & & 0.00 & 0.00 \\
\hline 1 & 285 & 111 & 189 & 81 & 15 & & & & & & & 0.88 & -1.39 \\
\hline 2 & 159 & 105 & 76 & 62 & 20 & 1 & & & & & & 0.78 & -1.94 \\
\hline 4 & 85 & 111 & 24 & 28 & 21 & 9 & 1 & 2 & & & & 1.04 & 0.26 \\
\hline 6 & 45 & 105 & 4 & 7 & 15 & 12 & 4 & 2 & 1 & & & 0.78 & -1.04 \\
\hline 8 & 50 & 173 & 1 & 3 & 11 & 13 & 11 & 7 & 1 & & 3 & 0.85 & -0.74 \\
\hline
\end{tabular}

From the $u$ values obtained for both tables and for all given doses, the distribution of unstable CA follows the Poisson distribution. The next step was the construction of doseeffect calibration curves based on data reported in the previous tables. Figure 10 presents the two curves. Considering the statistical error, the two curves overlap. This means that the time spent between fusion and irradiation (10 h and $24 \mathrm{~h})$ does not affect the number of CA created. Moreover, this confirms for the first time using PCC in combination with FISH and PNA probes that all unstable CA are completed within $10 \mathrm{~h}$ after irradiation, and the number of dicentrics are the same even after $24 \mathrm{~h}$.

Experiments performed later confirm that the quadratic term $\beta \mathrm{D}^{2}$ of the linearquadratic equation of the dose-effect curve needs $8 \mathrm{~h}$ to be expressed, while the linear term $\alpha \mathrm{D}$ is formed in a very quick process. ${ }^{16}$ Once we confirmed that the calibration curve obtained with fusion $24 \mathrm{~h}$ after irradiation can be universally used, we proceeded, using the same considerations previously reported, to construct the calibration curve dose-effect with confidence limits at $95 \%$ used for an estimation of the uncertainty of the dose. The calibration curve is shown in Fig. 11. 


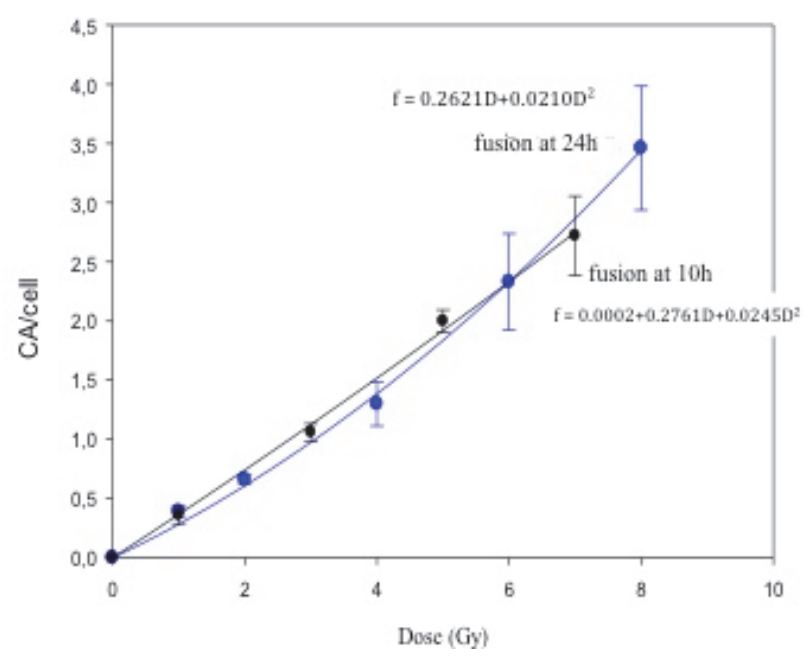

Fig. 10. Linear quadratic dose-effect curves of unstable CA yield in PCC's lymphocytes fused at $10 \mathrm{~h}$ and $24 \mathrm{~h}$ after irradiation with gamma rays from a ${ }^{60} \mathrm{Co}$ source. Unstable CAs are centromere and telomere painted using PNA probes. Error bars represent standard deviations of mean CA frequencies calculated from three independent experiments. $\mathrm{f}=\mathrm{CA}$ frequency, $\mathrm{D}=$ dose $(\mathrm{Gy})$.

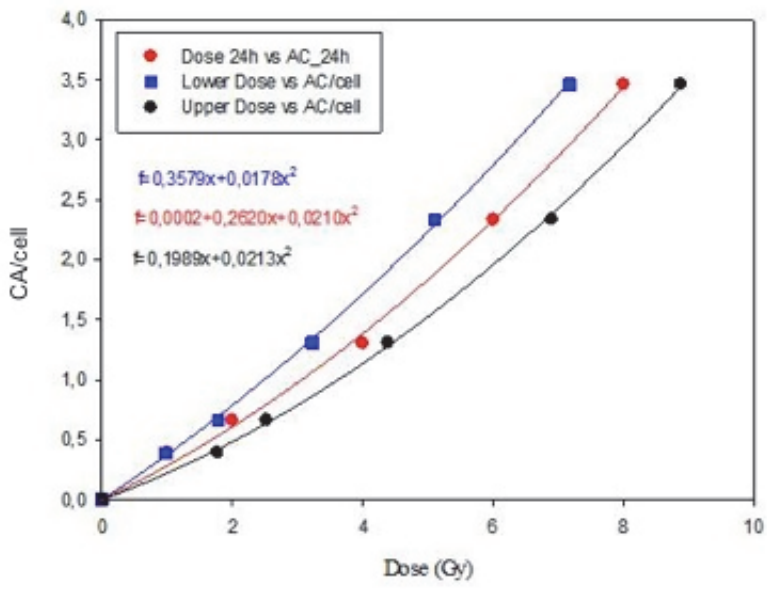

Fig. 11. Dose-effect curves representing unstable CA's frequency with 95\% upper and lower confident limits used to evaluate the uncertainty of the given dose in PCC's lymphocytes fused at $24 \mathrm{~h}$ after irradiation. $\mathrm{f}=\mathrm{CA}$ frequency, $\mathrm{x}=\operatorname{dose}(\mathrm{Gy})$.

Linear-quadratic dose response curves from manually scoring dicentrics and centric rings were obtained using different cytogenetic methods. The DCA is the "gold standard" of biological dosimetry, but the time required to obtain metaphases remains the major obstacle. The PCC fusion technique very rapidly induces premature condensation chromosomes in interphase and, counting excess from 46 fragments, is one of the most efficient and reliable methods if irradiated blood is available within $24 \mathrm{~h}$ from irradiation. 
The PCC technique using FISH for centromere and telomere staining with PNA probes has been shown to be a rapid and reliable method of scoring dicentrics and rings in interphase cells. It is an extremely elegant method of detecting primary DNA damage in interphase nuclei, but it is still considered technically challenging, time consuming in the scoring, and difficult to apply. Thus, only a few specialists in few laboratories use it.

IV) Testing the goodness of the calibration curves constructed for evaluation of absorbed gamma dose after an accidental irradiation

In order to test the accuracy of the constructed calibration curves and determine which assay is more suitable for triage biodosimetry, a simulation of a real accident was performed using blood samples that were irradiated with ${ }^{60} \mathrm{Co}$ gammas at two different doses of 1 Gy and 3 Gy and then blindly coded. The DCA assay was performed, and the chromosomes at metaphase were stained either with Giemsa or with FISH and PNA probes for centromeres and telomeres. Moreover, the PCC assay was performed and the chromosomes in interphase were stained as were those in metaphase. The yields of CA, Giemsa stained excess PCC fragments, and dicentrics plus centric rings in lymphocyte PCCs visualized by the FISH technique were obtained scoring 20 or 50 cells. Using the calibration curves and the yields obtained from this analysis, dose evaluations were performed for each method.

The results presented in Table 5 show the dose estimates obtained with different methodologies following blood sample irradiation at two different doses, 1 Gy and 3 Gy. DCA using FISH and PNA probes is shown to be a reliable, precise, accurate, and fast method for CA analysis, considering the time spent to score a metaphase (less than $20 \mathrm{~s}$ ). FISH with PNA probes analysis of CA in non-stimulated lymphocytes PCC is shown to be really advantageous because it is faster, sensitive, easy to score and accurate. Nevertheless, especially in the case of triage dosimetry where a fast response is needed and blood is available within $24 \mathrm{~h}$ from the irradiation, the analysis of Giemsa stained excess PCC fragments seems to be the easiest, fastest and most accurate method for absorbed dose assessment.

Table 5. Absorbed dose evaluation using the calibration curves of number of CA (dicentrics and centric rings) Giemsa staining, No of CA (dic and cR) FISH-PNA probes, excess PCC fragments Giemsa stained and scored immediately after exposure, CA in PCC spreads with FISH-PNA probes, constructed after whole body exposure at 1 Gy (A) and 3 Gy (B) irradiation for triage biodosimetry; scoring 50 cells were performed and mean doses with 95\% upper and lower confidence limits (UCL, LCL) are reported.

\begin{tabular}{|l|c|c|c|c|}
\hline $\begin{array}{c}\text { (A) } \\
\text { Exposure at 1 Gy }\end{array}$ & Giemsa (CA) & $\begin{array}{c}\text { FISH+PNA } \\
(\mathrm{CA})\end{array}$ & $\begin{array}{c}\text { PCC FISH+PNA } \\
(\mathrm{CA})\end{array}$ & $\begin{array}{c}\text { PCC+ Giemsa } \\
\text { (Excess fragments) }\end{array}$ \\
\hline $\begin{array}{l}\text { Scoring } \\
\text { (CA/ Excess fragments) }\end{array}$ & 8 & 11 & 17 & 54 \\
\hline Dose (Gy) & 1.6 & 1.2 & 1.1 & 1.1 \\
\hline LCL (Gy) & 1.2 & 1.0 & 0.8 & 0.8 \\
\hline UCL (Gy) & 1.9 & 1.3 & 1.4 & 1.4 \\
\hline
\end{tabular}


Table 5. (Continued)

\begin{tabular}{|l|c|c|c|c|}
\hline \multicolumn{1}{|c|}{$\begin{array}{c}\text { Exposure at 3 Gy } \\
\text { Explemsa (CA) }\end{array}$} & $\begin{array}{c}\text { FISH+PNA } \\
(\mathrm{CA})\end{array}$ & $\begin{array}{c}\text { PCC FISH+PNA } \\
(\mathrm{CA})\end{array}$ & $\begin{array}{c}\text { PCC+ Giemsa } \\
\text { (Excess fragments) }\end{array}$ \\
\hline $\begin{array}{l}\text { Scoring } \\
\text { (CA/ Excess fragments) }\end{array}$ & 33 & 35 & 38 & 143 \\
\hline Dose (Gy) & 3.3 & 2.9 & 2.6 & 3.1 \\
\hline LCL (Gy) & 2.8 & 2.4 & 2.0 & 2.8 \\
\hline UCL (Gy) & 3.7 & 3.1 & 3.1 & 3.4 \\
\hline
\end{tabular}

\section{Conclusion}

The goal of this work was to evaluate the received dose by the construction of calibration dose-effect curves using different cytogenetic techniques in case of an overexposure to gamma radiation due to an accident or terroristic event. The range of doses considered was from 0.3 Gy up to $8 \mathrm{~Gy}$. The time needed to give a reliable response on the absorbed dose is crucial, and this is especially true in emergency radiation medicine. Sophisticated, sensitive and reliable methodologies were applied for estimating the absorbed doses; the PCC technique, even though difficult to apply, overcomes the need of two days for lymphocytes to go to mitosis when compared with the analysis of dicentrics in metaphase. Due to the fact that PNA probes are relatively inexpensive, FISH analysis using centromere/telomere PNA probes can be used as a next-generation biological dosimetry, especially when combined with PCC for dicentric and centromere ring scoring. At the present time, the PCC assay based on excess fragment scoring when irradiated samples are available within $24 \mathrm{~h}$ cannot be replaced by other methodologies in terms of speed and accuracy, even with a relatively small number of scored cells.

\section{Acknowledgments}

The authors are immensely grateful to Dr. G. Pantelias and Dr. D. Terzoudi of N.C.S.R. "Demokritos", Athens Greece, who trained us on the methodologies used and for their contribution in discussions and comments on earlier versions of the manuscript.

\section{References}

1. Sr. J.W. Poston et al., Health Phys. 89, 450 (2005).

2. J.K. Timins et al., N. J. Med. 101 (9 Suppl), 66 (2004).

3. IAEA, Cytogenetic Dosimetry: Applications in preparedness for and response to radiation emergencies (International Atomic Energy Agency, Vienna, 2011).

4. K. Rothkamm et al., Radiat. Res. 180(2), 111 (2013).

5. L. Shi et al., Radiat. Res. 177, 533 (2012).

6. J. Meyne et al., Mutation Res. 226(2), 75 (1989).

7. P. Paulasova et al., Ann. Genet. 47(4), 349 (2004).

8. G.E. Pantelias and H.D. Maillie, Radiat. Res. 99, 140 (1984).

9. G.E. Pantelias, and H.D. Maillie, Somat. Cell Genet. 9, 533 (1983).

10. J.R.T. Savage, Curr. Top. Radiat. Res. 6, 129 (1970).

11. W. Merkle, Radiat. Environ. Biophys. 21, 217 (1983).

12. D.C Lloyd., R.J. Purrott and E.J. Reeder, Mutat. Res. 72523 (1980). 
13. M. Szluinska et al., Statistical methods for biological dosimetry, Health Protection Agency, Radiation Protection Division Report 011 (2005).

14. C. Beinke et al., Health. Phys. 98, 261 (2010).

15. R. M'kacher et al., Int. J. Radiat. Onc. (biology contribution) 91(3), 640 (2015).

16. R. Greinert et al., Radiat. Res. 144, 190 (1995). 